\title{
Virtual Fixtures for Robotic Cardiac Surgery
}

\author{
Shinsuk Park ${ }^{1}$ Ph.D., Robert D. Howe ${ }^{1}$ Ph.D., and David F. Torchiana ${ }^{2}$ MD \\ ${ }^{1}$ Harvard University, Division of Engineering and Applied Sciences, Cambridge, Mass., USA \\ \{sspark, howe\}@deas.harvard.edu \\ ${ }^{2}$ Massachusetts General Hospital, Division of Cardiac Surgery, Boston, Mass., USA
}

\begin{abstract}
We are developing virtual fixtures for the internal mammary artery (IMA) harvest portion of robot-assisted coronary artery bypass graft procedures. A preoperative CT scan will be processed to define the location of the IMA. In surgery, the patient's anatomy will be registered to the image data, then a virtual fixture will constrain the instrument's motions, as commanded by the surgeon, to appropriate paths adjacent to the artery. As a preliminary test, a virtual wall is implemented on a commercial surgical robot system. Results from a dissection task show that execution is faster and more precise than with conventional freehand techniques.
\end{abstract}

\section{Introduction}

Traditional methods in cardiothoracic surgery require large incisions such as sternotomies. Recent work has demonstrated that robotic systems enable execution of coronary artery bypass graft (CABG) procedures through small incisions [1]. In practice, these systems have proved cumbersome to use, with shortcomings that include decreased visual and haptic information, motion constraints, and the need for cognitive spatio-motor remapping from the surgeon's hands to the instrument space.

We propose to help alleviate these difficulties through virtual fixtures [2]. A virtual fixture is a computer-generated constraint that simplifies task execution by reducing precision requirements or the number of degrees of freedom that must be controlled. On a desktop computer, these fixtures are analogous to computer mouse features such as snap-to-grid that make it simpler to precisely position the cursor. In this study, the validity of the concept of virtual fixtures is tested on the ZEUS surgical robot system (Computer Motion, Inc., Goleta, Calif.). We report the results of in vitro testing of a virtual wall fixture for a blunt dissection task that prevents the surgical instrument from entering a specified volume of tissue.

\section{Methods}

This project focuses on robot-assisted minimally-invasive coronary artery bypass graft $(\mathrm{CABG})$ procedures. In this procedure, the interior mammary artery (IMA) is mobilized from the interior chest wall, and one end is attached to the coronary arteries to provide a new blood supply for the heart. Approximately $10-20 \mathrm{~cm}$ are dissected free, using blunt dissection and a harmonic scalpel or electrocautery. In the minimally invasive robotic approach, this is a laborious process that can occupy the majority of the operating time, over an hour in some cases. This study focuses on development of virtual fixtures for the IMA harvest portion of robot-assisted CABG procedures. 
In the planned procedure, the patient will undergo a CT scan with arterial contrast agent before surgery. In initial animal tests, metal pins will be inserted between the ribs to provide fixed landmarks during imaging and surgery. The resulting image set will be processed to define the location of the artery relative to the registration pins. During the procedure, the surgeon will bring the tip of a robot-mounted instrument into contact with each pin to establish registration; future implementations will use landmark-based registration. A virtual fixture will constrain the instrument's motions, as commanded by the surgeon, to appropriate dissection planes adjacent to the artery. This reduces precision requirements on the surgeon.

We have implemented virtual fixtures on the ZEUS surgical robot system. The complete system comprises two instrument positioner robot arms controlled by the surgeon in manual teleoperation mode, and a third endoscope positioner. The virtual fixture uses a single instrument positioner. To assess the performance benefits of a virtual fixture, we compared blunt dissections with and without the aid of a virtual wall. The computer-generated virtual wall confines the slave instrument tip to the region outside a specified plane. The slave instrument is free to follow the master instrument in all three dimensions outside the proscribed region, while it follows only lateral motions on the wall surface if the master controller is moved within the region.

Four subjects ( 2 surgeons and 2 engineers) were instructed to dissect a $2 \times 1.5 \times 1 \mathrm{~cm}$ segment from a block of soft material simulating tissue, but to avoid penetrating the wall, as marked with lines on the material. The subjects were seated in front of a video monitor providing an image of the operative site, and manipulated the master controller as in the unmodified system. Performance measures include time to completion and number of excursions into the wall beyond a safety margin of $5 \mathrm{~mm}$.

\section{Results and Discussion}

The effect of the fixture on task performance is summarized in Tables 1 and 2. The virtual wall reduced completion time by over $27 \%$ and eliminated excursions beyond the desired region, emphasizing the safety benefits of using the fixture.

Implementation of the image processing, registration, and surgeon interface portions of the system are underway. We are examining issues of registration accuracy, including instrument deflection and tissue movement between imaging and procedure.

Table 1. Time to completion (sec).

\begin{tabular}{c|cccc|c} 
Virtual & \multicolumn{4}{|c|}{ Subject } & Means \\
\cline { 2 - 5 } Fixture & 1 & 2 & 3 & 4 & \\
\hline OFF & 211 & 472 & 223 & 321 & 307 \\
ON & 173 & 345 & 155 & 215 & 222
\end{tabular}

Table 2. Number of excursions.

\section{References}

1. Stephenson, E. R. Jr, Sankholkar, S., Ducko, C. T., Damiano, R. J. Jr: Robotically assisted microsurgery for endoscopic coronary artery bypass grafting. Ann. Thoracic Surg. 66 (3) (1998) 1064-1067.

2. Rosenberg, L. B.: Virtual fixtures: Perceptual Tools for Telerobotic Manipulation. In: Proc. IEEE Virtual Reality Annual Intl. Symposium (1993) 76-82. 